\title{
Intervenciones de enfermería del campo fisiológico complejo en unidades de Cuidado Intensivo
}

\section{Complex psychological intervention by nurses in intensive care units}

\author{
Ingri Lizeth Sarmiento', Diana Mileth Tovar Medina', \\ Fabián Horacio Olaya Forero', Dolly Arias Torres²
}

\begin{abstract}
Resumen
Objetivo: Contrastar el nivel de conocimientos de los enfermeros de una Institución Prestadora de Servicios de Salud de IV Nivel respecto a las intervenciones no delegables del campo fisiológico complejo con los estandarizados en este servicio. Materiales y Métodos: Estudio descriptivo, cuantitativo y prospectivo. Participaron ocho enfermeras de la $\mathrm{UCl}$ adultos; la información se recolectó a través de encuesta y observación directa con ayuda de cuestionario auto diligenciado y guía de entrevista. Análisis estadístico descriptivo obtenido a través del programa SPSS versión 15. Resultados: La tercera parte de los profesionales de enfermería obtuvieron nivel de conocimiento medio y alto mientras que $25 \%$ registraron nivel bajo. Por otro lado, el nivel de delegación fue $44.64 \%$, asociada con la primera variable. Conclusión: se encontró un nivel de conocimiento medio y alto respecto a las intervenciones del campo fisiológico complejo y una delegación del $44.6 \%$.
\end{abstract}

Palabras clave: Enfermería, Intervenciones del campo fisiológico complejo, UCI. Procesos de Enfermería/Clasificación; Diagnóstico de Enfermería; Cuidados Intensivos; Sistemas de Información en Hospital

\begin{abstract}
Objective: Contrast the level of expertise of nursing staff in an institution providing level IV health care in regard to non-delegable complex psychological interventions with the standards. Method: A descriptive, quantitative and prospective study. Eight nurses from the adult ICU participated. The information was collected through surveys and direct observation with the help of a self-completed questionnaire and an interview guide. The descriptive statistical analysis was obtained using SPSS, version 15. Results: One third of the nursing staff showed a medium and high level of expertise, whilst $25 \%$ had a low level. On the other hand, the level of delegation was $44.64 \%$ associated with the first variable. Conclusions: The study found a medium and high level of expertise regarding complex psychological interventions and a delegation rate of $44.6 \%$.
\end{abstract}

Key words: Nursing; Complex Psychological Interventions; ICU; Nursing/Classification Processes; Nursing Diagnostics; Intensive Care; Information Systems in Hospitals.

\section{Introducción}

Las intervenciones de enfermería son tratamientos dirigidos a la persona, la familia y la comunidad, basados en el conocimiento y juicio crítico, realizados para favorecer el resultado esperado de los pacientes. Incluyen cuidados directos, indirectos y en colaboración, en aspectos fisiológicos, psicológicos y social. ${ }^{[1]}$

Según métodos de análisis de semejanzas, agrupaciones jerárquicas, juicio clínico y revisión de expertos, en 2009, se

1. Enfermeros Especialistas en Cuidado Crítico, Universidad Surcolombiana de Neiva. Colombia.

2. Ph.D., Profesora Titular Programa de Enfermería. Universidad Surcolombiana de Neiva. Colombia, correo electrónico: dolaria@usco.edu.co

Recibido: 29/04/2014- Revisado: 16/05/2015-Aceptado: 28/06/2015 
clasificaron 542 intervenciones de Enfermería organizadas en siete campos: fisiológico básico, fisiológico complejo, conductual, seguridad, familia, sistema sanitario y comunidad. En este estudio se abordaron las del segundo grupo[1]; ello en atención a que en estudios previos se encontró que el mayor porcentaje de intervenciones pertenece al campo fisiológico complejo. ${ }^{[2-4]}$

Las intervenciones del campo físiológico complejo son los cuidados que apoyan la regulación homeostática, competencia profesional fundamental en las Unidades de Cuidado Intensivo (UCI); se subdividen en intervenciones para el Control de ácido base y electrólitos, fármacos, neurológico, respiratorio, piel /heridas y perfusión tisular, Cuidados perioperatorios y Termorregulación. En total suman 180, de las cuales 168 son "no delegables". [1]

Las intervenciones no delegables son aquellas, que, con base en el análisis de las circunstancias de tiempo, modo y lugar lo mismo que por la complejidad de la situación de salud de las personas, deben ser asumidas por el profesional de enfermería, quien fundamenta su práctica en teorías y tecnologías propias y en conocimientos actualizados de las ciencias biológicas, sociales y humanísticas ${ }^{[5]}$.

Pese a existir regulación sobre definición y funciones específicas del profesional de enfermería, con frecuencia se encuentra delegación de procedimientos específicos a personal sin la suficiente preparación, acto que atenta contra el desempeño profesional y trasgrede reglamentación nacional e internacional vigente.

En exploración realizada en febrero de 2013 a Coordinadores de las ocho unidades de cuidado intensivo de la ciudad de Neiva, se evidenció que los procedimientos invasivos delegados con mayor frecuencia son: canalización de vena periférica, transfusión de hemoderivados y administración de medicamentos. En menor proporción se encontró el cateterismo nasogástrico y vesical. (Resultados no publicados).

Por lo anteriormente expuesto, se consideró importante realizar este trabajo con el objetivo de describir las intervenciones no delegables del campo físiológico complejo realizadas por los profesionales de Enfermería en una UCI de Neiva, el nivel de conocimientos sobre las mismas y las pautas de acción para que se cumpla con lo normado. Con los resultados se reflexionó sobre la importancia de que las enfermeras y enfermeros asuman lo de su incumbencia, factor determinante en la calidad del cuidado.

\section{Materiales y métodos}

Se realizó un estudio descriptivo, cuantitativo y prospectivo; participó una población constituida por 12 profesionales de enfermería, de éstos se incluyeron ocho que cumplían con los siguientes criterios: laborar en la unidad de cuidado intensivo en una IPS de cuarto nivel, con una experiencia mínima de tres meses en cuidado crítico. Se excluyeron: enfermeros con funciones administrativas ${ }^{[6]}$ influencia directa en el estudio.

Como instrumento de recolección de información se empleó la encuesta y la observación directa, mediante cuestionario auto diligenciado, lista de chequeo y guía de entrevista.

El primer instrumento, utilizado para la medición de conocimientos, se estructuró en dos partes, la primera con datos generales y la segunda con las intervenciones del campo fisiológico complejo no delegables. Por protocolo institucional, 39 de esas intervenciones son realizadas por médicos, fisioterapeutas o enfermeras de otros servicios con entrenamiento especial. Se adicionaron nueve intervenciones específicas de cuidado crítico para un total de 138 (Tabla 1).

Tabla 1. Número de intervenciones de Enfermería no delegables. UCI IPS IV Nivel. Neiva, 2013.

\begin{tabular}{lc}
\hline Clase & $\begin{array}{c}\text { No Intervenciones } \\
\text { no delegables }\end{array}$ \\
\hline Control ácido básico & 1 \\
Control de fármacos & 15 \\
Control neurológico & 12 \\
Cuidados perioperatorios & 11 \\
Control respiratorio & 5 \\
Control piel y heridas & 16 \\
Control Termorregulación & 7 \\
Control perfusión tisular & 47 \\
Específicas de Cuidado Crítico & 9 \\
\hline Total & 138 \\
\hline
\end{tabular}

En la observación directa se utilizó lista de chequeo con 138 intervenciones (Anexo B). Se realizaron 126 observaciones durante los días hábiles de octubre de 2013, entre las 07 y las 19 horas.

Después de procesar la información, fue presentada a los sujetos de estudio, con quienes se hizo un análisis crítico de lo encontrado y de los posibles factores asociados a los hallazgos.

El procesamiento de la información se hizo a través de Excel 2010 y el Software Estadístico SPSS versión 15.

\section{Resultados}

Nivel de conocimiento de los profesionales de enfermería de la uci respecto a las intervenciones no delegables del campo fisiológico complejo y las especificas de cuidado critico.

\section{El nivel de conocimiento fue alto, medio o bajo según escala (Tabla 2)}

$75 \%$ de los profesionales de enfermería obtuvieron nivel de conocimiento medio y alto, mientras que $25 \%$ registraron nivel bajo. Las intervenciones reconocidas en mayor proporción como no delegables, en orden descendente fueron: control ácido base y electrólitos, control de perfusión tisular 
Tabla 2. Matriz de valoración del nivel conocimiento de los profesionales de enfermería respecto a las intervenciones del campo fisiológico complejo y de las específicas de Cuidado Crítico en una UCI. IPS IV nivel de Neiva, 2013.

\begin{tabular}{ll}
\hline Nivel de conocimiento & criterio \\
\hline ALTO & Reconocimiento de más de 111 intervenciones no delegables. \\
MEDIO & Reconocimiento de 83 A 110 intervenciones no delegables. \\
BAJO & Reconocimiento de menos de 82 intervenciones no delegables.
\end{tabular}

y control respiratorio; en contraste, las de menor reconocimiento fueron: control de la termorregulación, de piel y heridas y cuidados perioperatorios. (Tabla 3)

Intervenciones nic del campo fisiológico complejo no delegables realizadas por los profesionales de enfermería en las unidades de cuidado intensivo adultos en la uci a. Ips iv nivel. Neiva, 2013

De las ciento treinta y ocho (138) intervenciones NIC no delegables que se evaluaron durante las observaciones realizadas en la Unidad de cuidado intensivo adulto de una IPS de IV nivel de Neiva, se encontró que setenta y siete (77) intervenciones se desarrollaron en el periodo de observación que corresponde al 55.8\%. Ocuparon el primer lugar las de perfusión tisular seguidas en orden descendente por las de control ácido base y electrolitos, control de fármacos, control de piel/heridas, control neurológico, específicas de UCI, control respiratorio, termorregulación y cuidados peri operatorios (Tabla 4).

De las 77 intervenciones observadas, fueron delegadas en menor proporción las de control neurológico, control acido base - electrolitos, control de fármacos y cuidados periopera-
Tabla 3. Distribución porcentual del nivel de conocimiento de los profesionales de enfermería de la $\mathrm{UCl}$ respecto a las intervenciones no delegables del campo fisiológico complejo y las específicas de cuidado crítico. IPS IV nivel. Neiva, 2013.

\begin{tabular}{lcc}
\hline Clase & SI* $^{*}(\%)$ & NO** (\%) \\
\hline Control ácido básico & 90.63 & 9.38 \\
Control perfusión tisular & 77.13 & 22.87 \\
Control respiratorio & 70 & 30 \\
Específicas de Cuidado Crítico & 68.06 & 31.94 \\
Control neurológico & 67.71 & 32.29 \\
Control de fármacos & 53.33 & 46.67 \\
Cuidados perioperatorios & 48.86 & 51.14 \\
Control piel y heridas & 47.66 & 52.34 \\
Control termorregulación & 37.5 & 62.5 \\
\hline
\end{tabular}

*Reconocimiento de las intervenciones como no delegable **No Reconocimiento de las intervenciones como no delegable

Tabla 4. Número de intervenciones NIC no delegables del campo fisiológico complejo y las específicas de cuidado critico realizadas en la UCI IV nivel. Neiva 2013.

\begin{tabular}{|c|c|c|c|c|c|c|c|}
\hline Clase & $\begin{array}{c}N^{\circ} \\
\text { Intervenciones } \\
\text { no delegables }\end{array}$ & $\begin{array}{c}\mathrm{N}^{\circ} \\
\text { intervenciones } \\
\text { realizadas }\end{array}$ & $\begin{array}{c}N^{\circ} \\
\text { repeticiones } \\
\text { de la intervención }\end{array}$ & $\begin{array}{c}N^{\circ} \\
\text { Delegada }\end{array}$ & Delegada & $x^{2}$ & $p$ \\
\hline Control ácido básico & 16 & 14 & 1070 & 81.78 & 18.22 & 375.5 & 0.0000001 \\
\hline Control de fármacos & 15 & 11 & 829 & 70.93 & 29.07 & 95.78 & 0.0000001 \\
\hline Control neurológico & 12 & 5 & 450 & 94.67 & 5.33 & 306.5 & 0.0000001 \\
\hline Cuidados perioperatorios & 11 & 2 & 43 & 69.77 & 30.23 & 3.638 & 0.02824 \\
\hline Control respiratorio & 5 & 4 & 140 & 0.00 & 100.00 & 178.2 & 0.0000001 \\
\hline Control piel y heridas & 16 & 8 & 817 & 20.32 & 79.68 & 477 & 0.0000001 \\
\hline Control termorregulación & 7 & 3 & 64 & 42.19 & 57.81 & 4.55 & 0.01646 \\
\hline Control perfusión tisular & 47 & 25 & 846 & 52.72 & 47.28 & 2.835 & 0.04612 \\
\hline \multicolumn{2}{|c|}{ Específicas de Cuidado Crítico 9} & 5 & 1212 & 38.86 & 61.14 & 171.6 & 0.0000001 \\
\hline Total & & 138 & 5471 & 55.36 & .64 & & \\
\hline
\end{tabular}


torios. Las más delegadas son las de control respiratorio, control de piel y heridas y las específicas de cuidado crítico. En general el nivel de delegación está por el orden del 44.64\%

Nivel de conocimiento y de delegación de intervenciones del campo fisiológico complejo por parte de profesionales de enfermería en una unidad de cuidado intensivo adultos. Ips iv nivel. Neiva, 2013

Se encontró alto nivel de conocimiento y baja delegación en control ácido base y electrolitos; en cuidados perioperatorios y perfusión tisular el nivel de conocimientos y la delegación se ubicaron en rango medio. Por otro lado, hubo nivel medio de conocimiento y baja delegación en Control neurológico; nivel medio de conocimiento y alta delegación en control respiratorio e intervenciones específicas de cuidado crítico; en control de piel y heridas y en termorregulación el nivel de conocimiento fue bajo y la delegación alta. En Control de fármacos se registró nivel bajo tanto en conocimientos como en la delegación. (Figura 1)

Posibles razones por las cuales se delegan las intervenciones del campo fisiologico complejo.

Luego de socializar los hallazgos con los enfermeros de la UCI se encontró que todos coinciden en que las principales causales de delegación son: sobrecarga laboral; alto número de pacientes, la mayoría de alta complejidad, baja relación enfermera paciente (1:7); déficit de profesionales para una atención de calidad y cumplimiento simultaneo de funciones administrativas. De la misma manera se identificaron las posi- bles implicaciones de tal delegación, a saber: ocurrencia de eventos adversos, demora en la atención, glosas, pérdidas de campos de práctica clínica y posibles demandas.

Los profesionales sugieren incrementar la relación enfermera-pacientes de acuerdo a estándares nacionales e internacionales $(1: 3,1: 4)$, programación de capacitaciones y/o reuniones en jornadas contrarias a la laboral y asignación de funciones exclusivamente asistenciales al profesional que brinda cuidado directo.

\section{Discusión}

Las teorías que fundamentan el conocimiento de enfermería, apoyadas y sustentadas en la práctica, orientan el desempeño profesional y facilitan la toma de decisiones en situaciones complejas. Esta razón motivó la valoración del nivel de conocimiento de las Intervenciones del campo fisiológico complejo, el cual fue alto y medio en $75 \%$.

Estudio realizado en Brasil encontró que 63,63\% de los enfermeros conocían la clasificación de las intervenciones de enfermería ${ }^{[7]}$; otro obtuvo una media de 5.48 , lo cual indica un nivel bajo ${ }^{[8]}$. La deficiente utilización de modelos conceptuales y teorías propias, interfieren en la autonomía profesional y en el desempeño independiente ${ }^{[9]}$; consideraciones consagradas en la Ley 266 de 1996, que define la Enfermería:

"como una profesión liberal y una disciplina de carácter social, cuyos sujetos de atención son la persona, la familia y lacomunidad, con sus características socio-

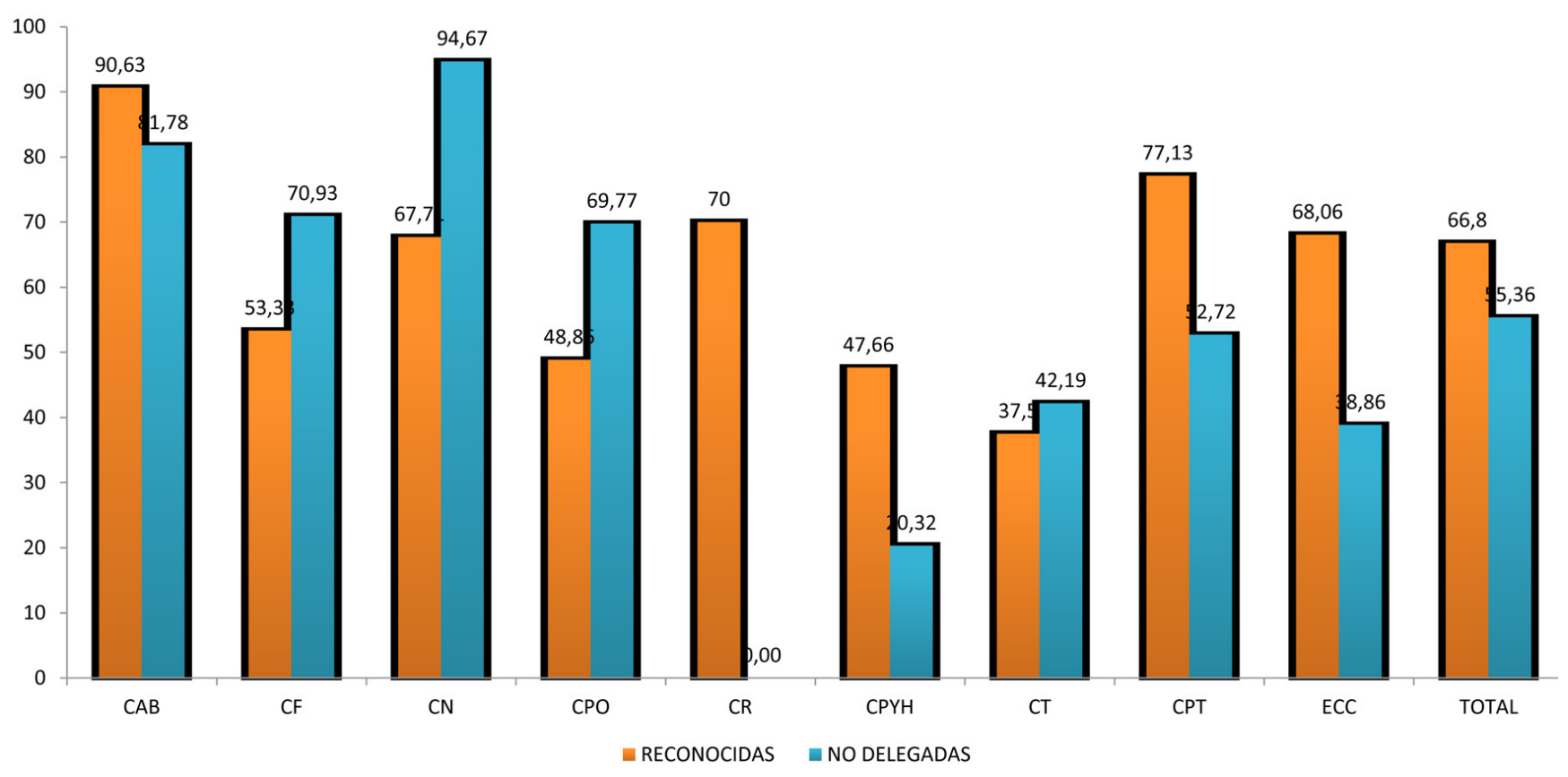

*CAB: Control Acido base, CF: control fármacos, CN: control neurológico, CPO: control perioperatorios, CR: control respiratorio, CPYH: control piel y heridas, CT: control termorregulación, CPT: control perfusión tisular, ECC: específicas de cuidado critico

Figura 1. Distribucion porcentual de intervenciones del campo fisiologico complejo identificadas como no delegables y de las realizadas por el profesional de enfermería. 
culturales, sus necesidades y derechos, así como el ambiente físico y social que influye en la salud y en el bienestar. Tiene como propósito general promover la salud, prevenir la enfermedad, intervenir en el tratamiento, rehabilitación y recuperación de la salud, aliviar el dolor, proporcionar medidas debienestar y contribuir a una vida digna de la persona. Fundamenta su práctica en los conocimientos sólidos y actualizados de las ciencias biológicas, sociales y humanísticas y en sus propias teorías y tecnologías. Su fin es dar cuidado integral de salud a la persona, la familia, la comunidad y a su entorno; ayudar a desarrollar al máximo los potenciales individuales y colectivos, para mantener prácticas de vida saludables que permitan salvaguardar un estado óptimo de salud en todas las etapas de la vida"[10].

Diferentes autores corroboran la importancia de la clasificación de las intervenciones de enfermería, pauta necesaria para el desarrollo del plan de atención según las pautas internacionales NIC, NANDA y $\mathrm{NOC}^{[2,11,12]}$; con el objetivo de mejorar la calidad del cuidado de enfermería ${ }^{[13]}$.

En las unidades de cuidado intensivo (UCI), las características de los pacientes hospitalizados y los equipos de alta tecnología, demandan del profesional de enfermería competencias cognitivas y procedimentales interdisciplinarias específicas para resolver e intervenir adecuadamente asuntos críticos del cuidado ${ }^{[14]}$. No obstante, ellos no encuentran aplicabilidad a la metodología NIC, NANDA y NOC en el área clínica; 78,57\% la utilizan en sus actividades de enseñanza y/ o investigación y sólo $18,18 \%$ la emplean en la práctica asistencial ${ }^{[7]}$.

Al parecer, la sobrecarga de trabajo asociada al alto número de pacientes asignados interfieren en la asunción de roles propios ${ }^{[4]}$ Además, en las funciones asignadas se prioriza la optimización del tiempo y los recursos de acuerdo con las exigencias institucionales, limitando el accionar profesional a la ejecución de intervenciones rutinarias que resuelven necesidades básicas; excluyendo asuntos de mayor complejidad a la hora de definir el plan de atención de Enfermería, [2, 4, 14, 15]. Estas observaciones son similares a las expuestas por los profesionales participantes en este estudio.

Escobar y cols, encontraron que el tiempo promedio para el desarrollo de las intervenciones de enfermería fue superior a 360 min en un turno de seis horas, lo cual indica déficit de profesionales y sobrecarga de trabajo[16]; hallazgo semejante se obtuvo en investigación que evidenció un déficit de $24 \%$ de enfermeros en relación al trabajo real que se genera, determinando así sobrecarga de trabajo en la atención directa y delegación de funciones ${ }^{[17,18]}$.

El nivel de delegación encontrado es análogo al registrado en investigación realizada en la misma institución pero en diferentes servicios (Resultados no publicados) ${ }^{[19]}$. La American Nurses Association (ANA) de fine la delegación como la transferencia de la responsabilidad pa ra la realización de una actividad de un individuo a otro manteniendo los resultados esperados ${ }^{[20]}$.
La delegación generada por el déficit de profesionales y la sobrecarga de trabajo promueven el aumento en la ocurrencia de eventos adversos, disminución de la calidad de la atención, glosas, prolongación de la estancia hospitalaria, mayor uso de antibióticos y aumento de los costos, entre $\operatorname{otros}^{[16,21,22]}$.

La delegación se ha generalizado que incluso en manuales de funciones de algunas instituciones se orienta a que las actividades no delegables del profesional sean realizadas por auxiliares de enfermería; al respecto, en revisión de estos documentos en las UCI de la ciudad de Neiva se encontró que intervenciones como la administración de medicamentos, la canalización de venas periféricas, y las transfusiones sanguíneas están dentro de las actividades que puede realizar el personal auxiliar, bajo una supervisión de los profesionales que es limitada por la sobrecarga laboral.

Esta problemática afecta a gran parte de los profesionales exponiéndolos a riesgos jurídicos, ya que la delegación no omite la responsabilidad del profesional de enfermería ${ }^{[5]}$, como se contempla en la legislación nacional

Con todo esto, se infiere que las instituciones prestadoras de servicios de salud deben cumplir con estándares nacionales e internacionales relacionados con la distribución de profesionales de enfermería según tipo de servicio, nivel de complejidad de la atención y situación de salud de los pacientes.

Actuar en concordancia mejora la calidad del cuidado y disminuye eventos adversos, pues está demostrado que la falta de personal suficiente y con la adecuada preparación tiene efectos inmediatos, a mediano y largo plazo: baja en la producción, calidad y seguridad de los servicios de enfermería, impacto sobre la eficacia de los equipos de salud y de la red asistencial y aumento de costos de la atención de salud ${ }^{[23]}$.

De igual manera es importante mejorar las condiciones de trabajo y brindar oportunidades de desarrollo profesional que redundan en beneficio institucional y responden a los procesos de habilitación y acreditación.

Es necesario replicar este ejercicio en las otras UCI de la ciudad y del departamento para justificar ante autoridades sanitarias la necesidad de ampliar el número de profesionales de Enfermería, condición indispensable para garantizar un cuidado de calidad.

\section{Conclusiones}

$75 \%$ de los profesionales de enfermería obtuvieron nivel de conocimiento medio y alto respecto a las intervenciones del campo fisiológico complejo no delegables, mientras que $25 \%$ registraron nivel bajo. Las reconocidas en mayor proporción, en orden descendente fueron: control ácido base y electrólitos, control de perfusión tisular y control respiratorio; en contraste, las de menor reconocimiento fueron: control de la termorregulación, de piel y heridas y cuidados perioperatorios.

El nivel de delegación fue de $44.64 \%$; fueron delegadas en menor proporción las intervenciones de control 
neurológico, control acido base-electrolitos, control de fármacos y cuidados perioperatorios. Las más delegadas son las de control respiratorio, control de piel y heridas y las específicas de cuidado crítico.

Principales causales de delegación identificadas: sobrecarga laboral; alto número de pacientes, la mayoría de alta complejidad, baja relación enfermera paciente (1:7); déficit de profesionales para una atención de calidad y cumplimiento simultaneo de funciones administrativas.

Posibles implicaciones de la delegación: ocurrencia de eventos adversos, demora en la atención, glosas, pérdidas de campos de práctica clínica y posibles demandas.

\section{Recomendaciones}

Replicar el estudio en otras UCI de la ciudad y del departamento para gestionar, ante autoridades sanitarias, el cumplimiento de estándares nacionales e internacionales relacionados con la distribución de profesionales de enfermería según tipo de servicio, nivel de complejidad de la atención y situación de salud de los pacientes.

\section{Referencias}

1. Bulechek, G.M., Clasificación de Intervenciones de Enfermería (NIC). 2009: Elsevier Health Sciences.

2. Lucena, A.d.F., et al., Nursing Interventions in the Clinical Practice of an Intensive Care Unit. Revista Latino-Americana de Enfermagem, 2010;18:873-880.

3. Salgado, P.d.O. and T.C.M. Chianca, Identification and mapping of the nursing diagnoses and actions in an Intensive Care Unit. Revista Latino-Americana de Enfermagem, $2011 ; 19: 928-935$.

4. Rojas, J.G. and P.P. Durango, Intensive care unit nursing care process application. 2010. 2010.

5. LEY 911 de 2004, in De los principios y valores eticos, del acto de cuidado de enfermeria, C.D.L.R.D. Colombia, Editor. 2004, Diario Oficial: Bogota.

6. Fàbregas, I.P. and G.J. i Solà, La profesión enfermera y la delegación. Ágora de enfermería, 2012. 16(4):181.

7. Almeida, M.d.A., A.K. Pergher, and D.F.d. Canto, Validation of mapping of care actions prescribed for orthopedic patients onto the nursing interventions classification. Revista Latino-Americana de Enfermagem, 2010;18:116-123.

8. May-Uitz, S., et al., Evaluación de conocimientos, habilidades y actitudes sobre el proceso de enfermería. Revista de Enfermería del Instituto Mexicano del Seguro Social: 13.

9. Gallardo, A.I., Evolución del conocimiento en enfermería. Medwave 2011 Abr;11(04):e5001 doi: 10.5867/ medwave.2011.04.5001, 2011.
10. LEY 266 DE 1996, in Por a cual se reglamenta la profesión de enfermería en Colombia y se dictan otras disposiciones, CONGRESO D.L.R.D.E. COLOMBIA, Editor. 1996, Diario Oficial No. 42.710, del 5 de febrero de 1996: Bogotá.

11. McCloskey, J. and G. Bulechek, Validation and coding of the NIC taxonomy structure. lowa Intervention Project. Nursing Interventions Classification. Image J Nurs Sch, $1995 ; 27(1): 43-9$.

12. Forneris, S.G. and C.J. Peden-McAlpine, Contextual learning: a reflective learning intervention for nursing education. Int J Nurs Educ Scholarsh, 2006. 3: Article 17.

13. Forbes, A., Clinical intervention research in nursing. Int J Nurs Stud, 2009;46(4):557-68.

14. Lake, S., C. Moss, and J. Duke, Nursing prioritization of the patient need for care: A tacit knowledge embedded in the clinical decision-making literature. International Journal of Nursing Practice, 2009;15(5):376-388.

15. BENEDET, S.A., A sistematização da assistência de enfermagem e as necessidades de cuidados de pacientes internados em terapia intensiva. 2012. Vol. 3.

16. Escobar Bermúdez, A.M.C., Jhon; Montenegro Rangel, Leidy paola; Vargas Zuñiga, Magda Lorena; Arias Torres, Dolly. Relación enfermera-o paciente según tiss28. Aplicacion clínica en Cuidado Intensivo. Revista facultad de salud-RFS. 2011. Vol 4, Num. 2:4-2;55-65.

17. Corredor, L.Y.S.S., Relación entre la sobrecarga laboral en enfermería y la incidencia de infecciones nosocomiales en la uci. 2008.

18. Romero-Massa, E., et al., Relación entre la carga laboral de enfermería y la gravedad del paciente en unidades de cuidado intensivo de adultos. 2011 . Vol. 11.

19. Gutiérrez Garzón, G.E.y.Q., Alexis Fernanda, Factores que influyen en el profesional de enfermería para delegar intervenciones clicas no delegables IPS de tercer nivel Neiva. 2012.

20. Torres Esperón, M., Metodología para definir funciones profesionales. Revista Cubana de Salud Pública, 2008; 34:1-12.

21. Herrejón, E.P. (2006) Volumen de pacientes atendidos en UCl y mortalidad. REVISTA ELECTRONICA DE MEDICINA INTENSIVA Artículo no 1008. Vol 6 nำ 7, julio 2006.

22. Alcaraz López, G., et al., Funciones del profesional de enfermería en salas de hospitalización de adultos: tratando de dar cuidado directo; Nursing professionals duties in adult hospitalization floors: trying to give direct care; Funções do profissional de enfermagem em salas de hospitalização de adultos: tratando de dar cuidado direto. Invest. educ. enferm, 2010;28(1):43-53.

23. Organization, W.H., Informe sobre la salud en el mundo: 2006: colaboremos por la salud. 2006. 